\title{
A CONNECTED COUNTABLE HAUSDORFF SPACE
}

\author{
R. H. BING
}

In Math. Ann. vol. 94 (1925) pp. 262-295, Urysohn gave an example of a connected Hausdorff space with only countably many points. Here is another.

EXAMPLE 1. The points of the space are the rational points in the plane on or above the $x$-axis. If $(a, b)$ is such a point and $\epsilon>0$, $(a, b)+\left\{(r, 0) \mid\right.$ either $\left|r-\left(a+b / 3^{1 / 2}\right)\right|<\epsilon$ or $\left.\left|r-\left(a-b / 3^{1 / 2}\right)\right|<\epsilon\right\}$ is a neighborhood.

To construct geometrically a neighborhood with center at $(a, b)$, consider an equilateral triangle with base on the $x$-axis and apex at $(a, b)$. (If $b=0$, regard $(a, b)$ as the triangle.) Then $(a, b)$ plus all rational points on the $x$-axis whose distances from a base vertex of the triangle are less than $\epsilon$ is an $\epsilon$-neighborhood with center at $(a, b)$.

This space satisfies the Hausdorff axioms and has the property that for each pair of neighborhoods, there is a point common to their closures. Hence, the space is connected.

Although this space has a countable basis, it is not regular and hence not metric. Its dimension depends on the definition of dimension used. In the Menger-Urysohn sense (the dimension is defined inductively in terms of boundaries of open sets) the space is one-dimensional, and in the Lebesgue sense (the dimension is defined in terms of orders of coverings) it is infinite-dimensional.

ExAmple 2. We may enlarge our description to get a connected countable Hausdorff space of any positive dimension (even infinite) in the Menger-Urysohn sense. We give one of dimension two.

The points of the space are the rational points of Euclidean 3 -space whose second and third coordinates are non-negative. If $(a, b, 0)$ is a point, an $\epsilon$-neighborhood with center at $(a, b, 0)$ is $(a, b, 0)$ $+\left\{(r, 0,0) \mid\right.$ either $\left|r-\left(a+b / 3^{1 / 2}\right)\right|<\epsilon$ or $\left.\left|r-\left(a-b / 3^{1 / 2}\right)\right|<\epsilon\right\}$. If $(a, b, c), c \neq 0$, is a point, an $\epsilon$-neighborhood with center at $(a, b, c)$ is the sum of $(a, b, c)$ and all $\epsilon$-neighborhoods with centers at points $(a, r, 0)$ where either $\left|r-\left(b+c / 2^{1 / 2}\right)\right|<\epsilon$ or $\left|r-\left(b-c / 2^{1 / 2}\right)\right|<\epsilon$.

THE UNIVERSITY OF WisCONSIN

Received by the editors August 15, 1952. 